\title{
Guide Flange Prosthesis for Management for a Hemimandibulectomy Patient- A Clinical Case Report
}

\author{
Dr. Laxmi Chhuchhar ${ }^{1}$, Dr. Mahesh A Gandhewar ${ }^{2}$ \\ ${ }^{1}$ Post graduate student, ${ }^{2}$ Professor and HOD \\ Department of Prosthodontics, A.C.P.M Dental College and Hospital, Dhule.-424001 Maharashtra, India.
}

\begin{abstract}
Prosthetic management of surgical defects has always been a big challenge for a prosthodontist. The sudden change in patient's perspective towards life affects the future outcome of any prosthetic rehabilitation. A good prosthesis can bring back the lost pride and smile on the patients face. A thorough understanding of post surgical anatomy and physiology is a prerequisite for the development of successful prosthesis. This case report describes prosthodontic management of a patient who has undergone hemi-mandibulectomy with a provisional prosthesis; modified mandibular guide flange prosthesis. The prosthesis helps patient moving the mandible normally, without deviation during functions like speech and mastication.
\end{abstract}

Key Words: Squamous cell carcinoma; Dental prosthesis design; Mandibular prosthesis; maxillofacial prosthesis; Segmental mandibulectomy, GFP. Guiding Flange Prosthesis.

\section{Introduction}

Since the sixteenth century acquired surgical defects have been restored by prosthetic replacements constructed from a variety of materials and techniques. The cosmetic, functional, and psychosocial results of oral cancer treatment may

Combine to produce devastating effects on patients, especially if the tumor is extensive or the treatment particularly aggressive. Indeed, oral cancer is noted for the toll it exacts from patients, from both the disease itself and the effects of its treatment. A variety of functions can be affected, including speech, deglutition, management of oral secretions, and mastication. Thus, maxillofacial prosthetic rehabilitation is a cornerstone of efforts to restore the head and neck cancer patient's oral functions and cosmetics following surgery to pretreatment baselines. Maxillofacial prosthetic therapy for acquired defects has become more complex and sophisticated with advances in surgical, physical and rehabilitative medicine. Acquired defects of the orofacial structures must be analyzed as to the specific cause and the consequent objectives of rehabilitation.

The prosthodontic rehabilitation of patient with acquired Mandibular defects is important as the balance and symmetry of Mandibular function is lost. There is an altered Mandibular movement and deviation of the residual fragment towards the defective side. This would hamper the functions of mastication and speech along with aesthetic and psychological comfort. The prosthodontic treatment modalities have made it possible to rehabilitate and manage patients with hemimandibulectomy defect. Guide flange prosthesis (GFP) is a mandibular conventional prosthesis designed for the patient who is able to achieve an appropriate mediolateral position of the mandible but is unable to repeat this position consistently for adequate mastication. ${ }^{2}$ This case report describes prosthodontic management of a patient who has undergone a hemimandibulectomy (from right condyle to left parasymphyseal region). A Modified GFP was fabricated to treat the patient.

\section{Case Report}

A 71 year old male patient with hemimandibulectomy done on his right side of the face reported to our clinic for prosthodontic rehabilitation of the deviated mandible. Patient's chief complaint was difficulty in mastication due to deviation of the mandible towards the defective side, thus causing disocclusion of the teeth on the normal side. Patient also complained of difficulty in speech and swelling. . A detailed case history revealed that the patient was operated due to squamous cell carcinoma of the right side of mandible 45 years back and had undergone radiation therapy postoperatively for a period of a year after that.. Extraoral examination shows facial asymmetry due to depression on righ side and deviation of the mandible towards the right. (Figure1).

The patient was able to achieve an appropriate mediolateral position of the mandible but was unable to repeat this position consistently for adequate mastication. On the basis of clinical and radiographic examination the patient was classified as Class II (severely compromised) according to Prosthodontic Diagnostic Index Resources for partial edentulous patients as described by McGarry et $\mathrm{al}^{4}$ and as class III (Lateral resection of the mandible with distal to the cuspid) according to classification of surgical impairment (cantor and curtis) ${ }^{5}$ (figure 2.) 
A stainless steel stock edentulous tray (modified by trimming buccal flange of right half) and irreversible hydrocolloid (Dentalgin; Prime dental products, Mumbai, India) were used to record preliminary impression of the mandibular arch. Maxillary impression was also made with irreversible hydrocolloid. The impressions were poured with Type III gypsum material (Kalstone; Kalabhai Karson, Mumbai, India) and casts were retrieved. A 19 gauge hard, round, stainless steel orthodontic wire (KC Smith and Co, Monmouth, UK) was manipulated (as shown in Figs 3) to fabricate a substructure for the modified GFP. The vestibular (buccal and lingual) flanges and the mandibular guide-flange were waxed-up with modeling wax (Modeling wax; Deepti Dental Products, Ratnagiri, India) around the wire substructure by keeping a maxillary cast in occlusion and subsequently acrylized into the clear heat-polymerized acrylic resin (DPI Heat cure clear; Dental products of India, Mumbai, India) to make the GFP (Fig. 4 ).

The GFP was tried in patient's mouth and the initial stability and retention was checked. The inclination of the guide-flange was adjusted by selectively trimming the teeth-contacting surface or adding the auto-polymerizing clear acrylic resin (DPI Cold cure clear; Dental products of India, Mumbai, India). Thus the smooth gliding flange surface was developed intraorally to guide the mandible in a definite closing point (rather than the area) in occlusion. Care should be taken to preserve the buccal- surface indentations of the opposing maxillary teeth which were guiding the mandible in a final definite closing point during mastication. The flange height was adjusted in such a way that it guided the mandible from large opening position (in practical limits of the height of the buccal vestibule) to the maximum intercuspation in a smooth and unhindered path. The prosthesis was delivered and post-insertion instructions were given (figure 5.). The patient was followed up at the regular interval of two months for next one year. Patient's last recall visit was after one and half year of the reconstruction. The patient was pleased with the overall performance of the prosthesis and successfully speaks and masticates without clinically significant deviation. ${ }^{6}$

\section{Discussion}

Depending upon the location and extent of the tumor in the mandible, various surgical treatment modalities like marginal, segmental, hemi, subtotal, or total mandibulectomy can be performed. ${ }^{3}$ Loss of mandibular continuity causes deviation of remaining mandibular segment(s) towards the defect and rotation of the mandibular occlusal plane inferiorly. Mandibular deviation toward the defect side occurs primarily because of the loss of tissue involved in the surgical resection. ${ }^{3}$ As we see in this first type of prosthesis ,a vertical extension from the buccal aspect of a mandibular prosthesis extends to contact the buccal surface of the opposing maxillary teeth. This extension maintains the mandible in the proper mediolateral position for vertical chewing, but little, if any, lateral movement is possible. Recent advancements in facial reconstructive surgery and osseointegrated dental implants provide a treatment modality that may adequately rehabilitate oral cancer patients so that they can return to a healthy, productive life. Though osseointegrated dental implants is the final solution for replacing the missing teeth for reconstructed mandibulectomy patients, the clinicians must wait for extensive period of time (more than a year) for completion of healing and acceptance of the osseous graft. Our principal aim was to maintain the esthetics during mandibular movements. Hence the GFP was fabricated in clear acrylic resin and the retentive wire components were kept distal to the mandibular canine to minimize the prosthesis display. Support for the GFP is no different from that of any other removable prosthesis, the natural teeth and the residual alveolar ridge being the primary sources. Multiple retentive clasps in widely distributed areas of the arch would be the best approach, but actual placement would be determined by the position of the teeth. Retentive elements should be no more rigid than necessary, but they require a more rigidity with a decreasing number of teeth. In the presented case retentive components were modified and incorporated into the prosthesis as a wire substructure. The buccal and lingual vestibular flanges can be brought closer by bending the occlusal cross-over wire components with a universal orthodontic plier to improve the retention.

The GFP can be regarded as a training type of prosthesis. If the patient can successfully repeat the mediolateral position, the GFP can often be discontinued. Some patient, however, may continue indefinitely with a guide flange, and the stress generated to the remaining teeth must then be carefully monitored.

\section{Conclusion}

A comfortable mandibular alignment is not always maintainable in the restoration of the patients with partially resected mandible. The guiding appliances can be a useful adjunct to preserve the mandibular function after partial mandibulectomy procedure to minimize associated complications like mastication, speech, and swelling as "Every human has the Devine right to look human". 


\section{References}

[1]. Beumer J, Curtis TA, Mark T. Marunick: MaxillofacialmRehabilitation - Prosthodontic and Surgical Considerations.

[2]. Desjardins RP. Relating examination findings to treatment procedures. In: Laney WR. Maxillofacial prosthetics. Littleton; PSG Publishing; 1979. p. 69-114.

[3]. Taylor TD. Diagnostic considerations for prosthodontic rehabilitation of the mandibulectomy patient. In: Taylor TD, editor. Clinical maxillofacial prosthetics. Chicago; Quintessence Publishing; 2000. p. 155-70.

[4]. McGarry TJ, Nimmo A, Skiba JF, Ahlstrom RH, Smith CR, Koumjian JH, Arbree NS. Classification system for partial edentulism. J Prosthodont 2002;11:181-93.

[5]. Cantor R, Curtis TA. Prosthetic management of edentulous mandibulectomy patients -Part II, Clinical Procedures J Prosthet Dent $1971 ; 25 ; 546-55$

[6]. Pravinkumar Gajanan Patil Guide flange prosthesis for early management of reconstructed hemimandibulectomy: a case report J Adv Prosthodont 2011;3:172-6

[7]. M Satish , Nirmala Pasam Training flange appliance after hemimandibulectomy - A case report. SRM University journal of dental sciences. Vol 2, Issue4 . October- December 2011

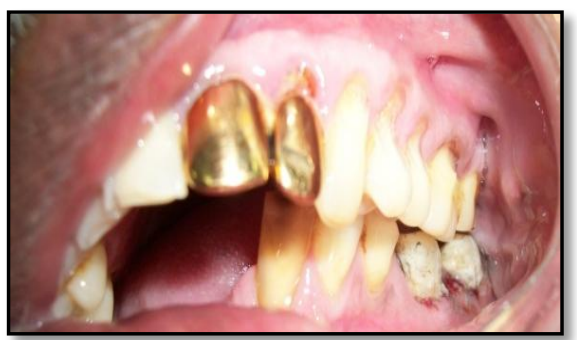

Figure: 1 Dissoclusion on normal side.

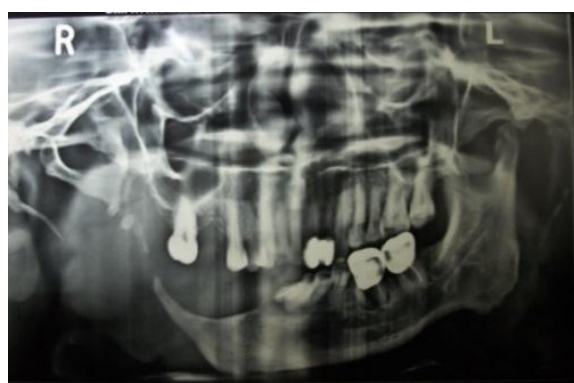

Figure 2: Post-surgical panoramic radiograph showing showing extensive radiolucent area in right mandible

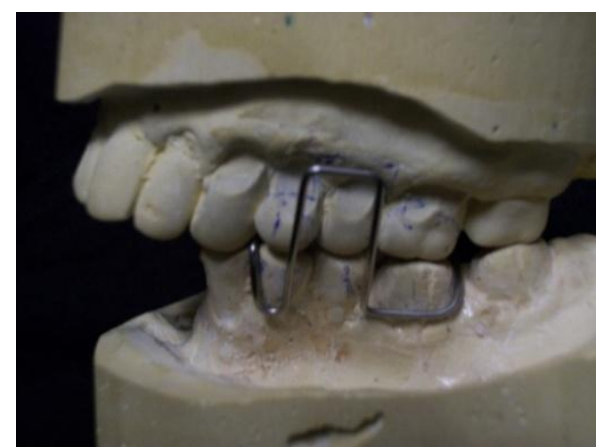

Figure 3 : Wire substructure :Buccal view.

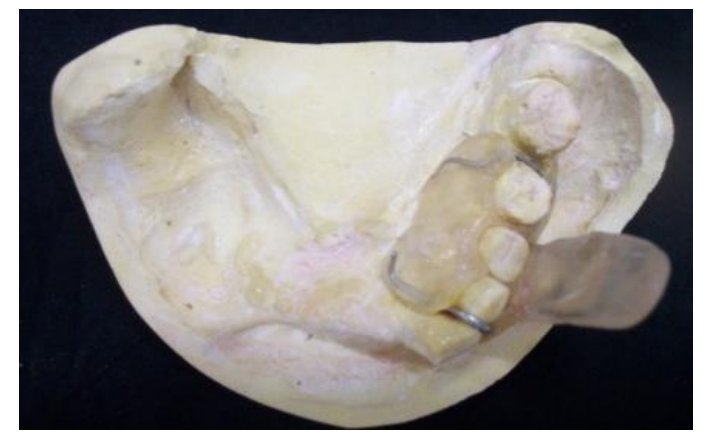

Figure 4 Completed Modified Guide flange prosthesis

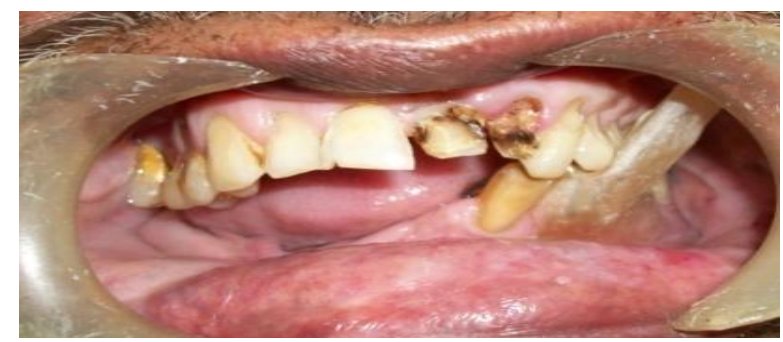

Fig.5 Prosthesis in place during definite closing point. 